\title{
Collisional Energy Loss of Fast Charged Particles in Relativistic Plasmas
}

\author{
Markus H. Thoma* \\ Theory Division, CERN, CH-1211 Geneva 23, Switzerland
}

(November 16, 2018)

\begin{abstract}
Following an argument by Kirzhnits we rederive an exact expression for the energy loss of a fast charged particle in a relativistic plasma using the quantum field theoretical language. We compare this result to perturbative calculations of the collisional energy loss of an energetic electron or muon in an electron-positron plasma and of an energetic parton in the quark-gluon plasma.
\end{abstract}

Keywords: Energy loss; Relativistic plasmas; Thermal Field Theory

PACS numbers: $52.60+\mathrm{h}, 12.38 . \mathrm{Mh}, 11.10 . \mathrm{Wx}$

*Heisenberg fellow 
The energy loss of a fast charged particle in a medium is a well studied subject [ []]. Recently the energy loss of energetic particles, such as leptons and partons, in relativistic plasmas has attracted great interest. In relativistic heavy ion collisions the energy loss of a high energy quark or gluon coming from primary hard collisions in the fireball may serve as a signature for the quark-gluon plasma formation [2]. In Supernovae explosions the energy loss of neutrinos, having a weak charge, in the plasma surrounding the stellar core might be an important mechanism for triggering the explosion [3].

The total energy loss of a particle in a medium can be decomposed into a collisional and a radiative contribution. While the first one originates from the energy transfer to the medium particles, the latter one is caused by radiation from the fast particle. Here we want to consider only the collisional component. Whereas the radiative energy loss dominates in the case of partons or charged leptons [4], the collisional one is dominant for neutrinos in a Supernova plasma due to the small coupling of the neutrinos to the medium.

In quantum field theory the collisional energy loss per unit length is defined as [5]

$$
\frac{d E}{d x}=\frac{1}{v} \int d \Gamma \omega,
$$

where $v$ is the velocity of the incident particle with energy $E$ and $\omega=E-E^{\prime}$ the energy transfer to the medium. The interaction rate $\Gamma$ can be calculated either from the matrix element of the process responsible for the energy loss or equivalently from the imaginary part of the self energy of the particle with four momentum $P=(E, \mathbf{p})$, and mass $M,(p=|\mathbf{p}|)$ [5]

$$
\Gamma(E)=-\frac{1}{2 E}\left[1-n_{F}(E)\right] \operatorname{tr}[(\not P+M) \operatorname{Im} \Sigma(E, p)],
$$

where $n_{F}(E)=1 /[\exp (E / T)+1]$ is the Fermi distribution in the case of a fermion propagating through a plasma of temperature $T$. In the following we restrict ourselves first to electrons or muons with high energies $E \gg T$ in an electron-positron plasma. Furthermore we assume first only small momentum and energy transfers, $\omega, k \ll T$. Assuming a one-loop approximation for $\Sigma$ but allowing for the most general photon propagator, indicated by the blob in Fig.1, we find [5]

$$
\left(\frac{d E}{d x}\right)_{\text {soft }}=\frac{e^{2}}{2 \pi v^{2}} \int_{0}^{k^{*}} d k k \int_{-v k}^{v k} d \omega\left[1+n_{B}(\omega)\right]\left[\rho_{l}(\omega, k)+\left(v^{2}-\frac{\omega^{2}}{k^{2}}\right) \rho_{t}(\omega, k)\right],
$$

where $k^{*} \ll T$ is the separation scale, decomposing the energy loss into a soft and a hard part. $n_{B}(\omega)=1 /[\exp (\omega / T)-1]$ is the Bose distribution and $\rho_{l, t}$ are the spectral functions of the full photon propagator, defined as

$$
D_{l, t}\left(k_{0}, k\right)=\int_{-\infty}^{\infty} d \omega \frac{\rho_{l, t}(\omega, k)}{k_{0}-\omega+i \varepsilon} .
$$

At finite temperature the photon propagator has only two independent components [6], given in Coulomb gauge by the longitudinal and transverse propagators [2]

$$
\begin{aligned}
& D_{l}\left(k_{0}, k\right)=\frac{1}{k^{2}-\Pi_{l}\left(k_{0}, k\right)+i \varepsilon}, \\
& D_{t}\left(k_{0}, k\right)=\frac{1}{k_{0}^{2}-k^{2}-\Pi_{t}\left(k_{0}, k\right)+i \varepsilon},
\end{aligned}
$$


where $\Pi_{l, t}$ are the longitudinal and transverse components of the polarization tensor. It should be noted that the soft collisional energy loss, discussed here, follows according to (3) only from the exchange of one dressed space-like $\left(\omega^{2}-k^{2}<0\right)$ photon from the particle to the medium according to linear response theory. However, the medium particles may undergo further interactions. The physical process corresponding to the imaginary part of the self energy of Fig1. can be found by using cutting rules. An example is shown in Fig.2. There is no diagram, where to or more photons are emitted from the fast particle, as it is the case e.g. for bremsstrahlung. In the case of a neutrino, however, diagrams containing two gauge boson lines are suppressed anyway.

The spectral functions can be expressed by the imaginary part of the photon propagator according to

$$
\rho_{l, t}(\omega, k)=-\frac{1}{\pi} \operatorname{Im} D_{l, t}(\omega, k) .
$$

For soft energy transfers $\omega \ll T$, as we assumed above, the photon distribution can be expanded, leading to

$$
1+n_{B}(\omega) \simeq \frac{T}{\omega}+\frac{1}{2}
$$

Substituting (7) into (3) only the second term in (7) contributes since the spectral functions are odd functions of $\omega$ [7].

Alternatively the soft energy loss can also be derived from classical plasma physics arguments. It follows from the induced electric field of the fast charged particle in the plasma, which reacts on the incident particle by the Lorentz force, causing the energy loss [8]. This process is known as the Fermi density effect [1]. Introducing the dielectric functions of the medium, the soft energy loss can be written as 9

$$
\left(\frac{d E}{d x}\right)_{s o f t}=-\frac{e^{2}}{4 \pi^{2} v^{2}} \int_{0}^{k^{*}} d k k \int_{-v k}^{v k} d \omega \omega\left[k^{2} \operatorname{Im} \epsilon_{l}(\omega, k)^{-1}+\left(v^{2}-\frac{\omega^{2}}{k^{2}}\right) \operatorname{Im}\left(\omega^{2} \epsilon_{t}(\omega, k)-k^{2}\right)^{-1}\right] .
$$

This expression is equivalent to (3), since the dielectric functions are related to the polarization tensor via [9 11]

$$
\begin{aligned}
& \epsilon_{l}(\omega, k)=1-\frac{\Pi_{l}(\omega, k)}{k^{2}}, \\
& \epsilon_{t}(\omega, k)=1-\frac{\Pi_{t}(\omega, k)}{\omega^{2}}
\end{aligned}
$$

and therefore also to the spectral functions, which are given by the imaginary part of the propagators (5), by

$$
\begin{aligned}
& \operatorname{Im} \epsilon_{l}(\omega, k)^{-1}=-\pi k^{2} \rho_{l}(\omega, k), \\
& \operatorname{Im}\left(\omega \epsilon_{t}(\omega, k)-k^{2}\right)^{-1}=-\pi \rho_{t}(\omega, k),
\end{aligned}
$$

where only the discontinuous part of the spectral functions coming from the imaginary part of the polarization tensor contributes. 
Now we want to derive an exact result for the soft collisional energy loss using a generalized Kramers-Kronig relation and the asymptotic behavior of the dielectric functions. For this purpose we introduce the response function of the medium $R\left(k_{0}, k\right)=$ $R_{l}\left(k_{0}, k\right)+R_{t}\left(k_{0}, k\right)$, given by [12]

$$
\begin{aligned}
& R_{l}\left(k_{0}, k\right)=-\frac{1}{\epsilon_{l}\left(k_{0}, k\right)}, \\
& R_{t}\left(k_{0}, k\right)=\frac{k^{2}-k_{0}^{2}}{k^{2}-k_{0}^{2} \epsilon_{t}\left(k_{0}, k\right)} .
\end{aligned}
$$

Using (5) and (9) we obtain

$$
R\left(k_{0}, k\right)=-k^{2} D_{l}\left(k_{0}, k\right)+\left(k_{0}^{2}-k^{2}\right) D_{t}\left(k_{0}, k\right) .
$$

Replacing the spectral functions in (3) or the dielectric functions in (8) by the quantity $R$, making the substitution $k \rightarrow q=\sqrt{k^{2}-\omega^{2}}$, i.e. introducing the magnitude of the four momentum of the exchanged photon, and using $\operatorname{Im} R(-\omega)=-\operatorname{Im} R(\omega)$ we find

$$
\left(\frac{d E}{d x}\right)_{s o f t}=\frac{e^{2}}{2 \pi^{2}} \int_{0}^{q^{*}} d q q \int_{0}^{\infty} d \omega \omega \frac{\operatorname{Im} R\left(\omega, \sqrt{q^{2}+\omega^{2}}\right)}{q^{2}+\omega^{2}} .
$$

Here we restricted ourselves to ultrarelativistic particles, $v=1$, and $q^{*} \ll T$. Eq. (13) agrees with Ref. [12], if we replace there $Q^{2}$ by $e^{2} / 4 \pi$.

The response function $R$ fulfills the following Kramers-Kronig relation [12]

$$
R\left(k_{0}, k\right)=\tilde{R}+\frac{2}{\pi} \int_{0}^{\infty} d \omega \omega \frac{\operatorname{Im} R(\omega, k)}{\omega^{2}-k_{0}^{2}-i \varepsilon},
$$

which can be shown to be equivalent to the definition of the spectral functions (4), if we use $\rho_{l, t}(-\omega)=-\rho_{l, t}(\omega)$. Here $\tilde{R}=\lim _{k_{0} \rightarrow \infty} R\left(k_{0}, k\right) \sim \lim _{k_{0} \rightarrow \infty} 1 / k_{0}^{2}=0$. The relation (114) can be generalized to the so-called Leontovich relation [13] exploiting causality, from which one obtains [12,14]

$$
R\left(k_{0}, \sqrt{k^{2}+k_{0}^{2}}\right)=R_{\infty}+\frac{2}{\pi} \int_{0}^{\infty} d \omega \omega \frac{\operatorname{Im} R\left(\omega, \sqrt{k^{2}+\omega^{2}}\right)}{\omega^{2}-k_{0}^{2}-i \varepsilon},
$$

where $R_{\infty}=\lim _{k_{0} \rightarrow \infty} R\left(k_{0}, \sqrt{k^{2}+k_{0}^{2}}\right)$.

The $\omega$-integral

$$
I=\frac{2}{\pi} \int_{0}^{\infty} d \omega \omega \frac{\operatorname{Im} R\left(\omega, \sqrt{q^{2}+\omega^{2}}\right)}{q^{2}+\omega^{2}}
$$

appearing in the energy loss (13i) agrees with the integral on the right hand side of the Leontovich relation, if we replace $\omega$ by $i q$ and $\sqrt{k^{2}+\omega^{2}}$ by 0 , i.e. $k^{2}=q^{2}$, in (15). Therefore we can write [12]

$$
I=R(i q, 0)-R_{\infty}
$$


Since the longitudinal and the transverse dielectric functions are identical at zero momentum [15], $\epsilon_{l}\left(k_{0}, 0\right)=\epsilon_{t}\left(k_{0}, 0\right)$, we have $R(i q, 0)=0 . R_{\infty}$ is related to the high frequency and momentum limit of the dielectric functions, which agrees with the vacuum result $\epsilon_{l}=1$. For the transverse part we have to consider corrections to the vacuum value. From the second equation of (9) we get

$$
\lim _{k_{0} \rightarrow \infty} \epsilon_{t}\left(k_{0}, \sqrt{q^{2}+k_{0}^{2}}\right)=1-\frac{\omega_{0}^{2}}{k_{0}^{2}}
$$

where

$$
\omega_{0}^{2} \equiv \lim _{k_{0} \rightarrow \infty} \Pi_{t}\left(k_{0}, \sqrt{q^{2}+k_{0}^{2}}\right)
$$

Using the Kramers-Kronig relation for the transverse dielectric functions it can be shown [12], that $\omega_{0}$ is independent of $q$. It can be considered as the effective thermal mass of the transverse high frequency plasma excitations, which is given by $\omega_{0}^{2}=e^{2} n\langle 1 / \Omega\rangle$ in the relativistic limit [12]. Here $n$ is the number density of the medium and $\Omega$ the energy of the plasma particles. In the non-relativistic limit $\omega_{0}$ is identical to the plasma frequency [12].

Using (11) together with the high frequency and momentum limit of the dielectric functions we get

$$
I=-R_{\infty}=\frac{\omega_{0}^{2}}{q^{2}+\omega_{0}^{2}}
$$

Combining this result for $I$ with (13) we end up with

$$
\left(\frac{d E}{d x}\right)_{\text {soft }}=\frac{e^{2}}{4 \pi} \omega_{0}^{2} \ln \frac{q^{*}}{\omega_{0}},
$$

where we assumed $q^{*} \gg \omega_{0}$.

The unknown parameter $\omega_{0}$ following from the full transverse polarization tensor serves as an infrared cutoff for the photon exchange. Since the total collisional energy loss has to be independent of the arbitrary separation scale $q^{*}$, the hard part has to assume the form

$$
\left(\frac{d E}{d x}\right)_{h a r d}=\frac{e^{2}}{4 \pi} \omega_{0}^{2} \ln \frac{q_{\max }}{q^{*}},
$$

where $q_{\max }$ is proportional to the maximum energy transfer, i.e. $q_{\max } \sim \sqrt{E T}$ in the relativistic limit $E \gg \Omega$ [12], which we have considered here. Note that the hard contribution to the energy loss contains besides $t$-channel diagrams, as the one in Fig.2, also $s$ - and $u$ channel ones, which, however, do not contribute to the leading logarithm.

Hence we obtained a very simple expression

$$
\frac{d E}{d x}=\frac{e^{2}}{4 \pi} \omega_{0}^{2} \ln \frac{q_{\max }}{\omega_{0}}
$$

for the exact result of the collisional energy loss, independent of any approximation to the full photon propagator or the dielectric functions of the medium, respectively. To logarithmic accuracy the final result just depends on the parameter $\omega_{0}$. 
As an example we consider the high temperature limit of the energy loss. There it can be calculated to leading order perturbation theory using the Hard Thermal Loop (HTL) resummation technique [16]. Computing the soft energy loss using the HTL resummed photon propagator in Fig.1 and calculating the hard part from the tree level scattering matrix elements one finds in the limit $v=1$ [5]

$$
\frac{d E}{d x}=\frac{e^{2}}{4 \pi} \omega_{0}^{2}\left(\ln \frac{\sqrt{E T}}{\omega_{0}}+0.120\right),
$$

where $\omega_{0}^{2}=3 m_{\gamma}^{2} / 2$. The thermal photon mass $m_{\gamma}$, which is equivalent to the plasma frequency, is given by $m_{\gamma}=e T / 3$. Indeed $\omega_{0}$ is given by the high frequency and momentum limit (19) of the transverse HTL polarization tensor [17]

$$
\Pi_{t}^{H T L}\left(k_{0}, k\right)=\frac{3}{2} m_{\gamma}^{2} \frac{\omega^{2}}{k^{2}}\left[1-\left(1-\frac{k^{2}}{k_{0}^{2}}\right) \frac{k_{0}}{2 k} \ln \frac{k_{0}+k}{k_{0}-k}\right]
$$

confirming the general result (23) in the HTL limit. Also $\omega_{0}^{2}=e^{2} n\langle 1 / \Omega\rangle$ holds for the HTL case, where [18]

$$
m_{\gamma}^{2}=\frac{4 e^{2}}{3 \pi^{2}} \int_{0}^{\infty} d k k n_{F}(k)
$$

because

$$
n=4 \int \frac{d^{3} k}{(2 \pi)^{3}} n_{F}(k), \quad\left\langle\frac{1}{\Omega}\right\rangle=\frac{\int \frac{d^{3} k}{(2 \pi)^{3}} \frac{1}{k} n_{F}(k)}{\int \frac{d^{3} k}{(2 \pi)^{3}} n_{F}(k)} .
$$

In the case of the collisional energy loss of quarks or gluons in a quark-gluon plasma we simply have to replace the factor $e^{2}$ in (23) by $C_{F} g^{2}=4 g^{2} / 3$ for quarks and by $C_{A} g^{2}=3 g^{2}$ for gluons, where $g$ is the strong coupling constant. Furthermore $\omega_{0}^{2}$ is now the high frequency and momentum limit of the transverse gluon polarization tensor, which is given by $3 m_{g} / 2$, where the effective gluon mass reads $m_{g}^{2}=g^{2} T^{2}\left(1+n_{f} / 6\right) / 3$ in a QGP containing $n_{f}$ thermalized quark flavors. Using the HTL limit for $\omega_{0}$ in (23) we reproduce again the result obtained from an explicit HTL resummed calculation within the logarithmic approximation [19]. Another application of (23) has been discussed in Ref. [20] in connection with the neutrino energy loss in matter.

Summarizing, we have shown that different definitions of the collisional energy loss, based either on quantum field theory or on plasma physics, are equivalent. Translating the arguments, based on a generalized Kramers-Kronig relation, given by Kirzhnits [12] to a quantum field theoretical language, using self energies, propagators and their spectral functions, we gave an exact result for the collisional energy loss (23). Within the logarithmic approximation it contains the effective mass of the high frequency transverse plasma mode as the only parameter. Assuming that this mass is given approximately by the high temperature result, we obtain an simple estimate for the collisional energy loss for energetic electrons and muons in a QED plasma and for partons in the quark-gluon plasma. Finally we showed that the perturbative result for the collisional energy loss, obtained within the HTL resummation method, is in agreement with the general result found by Kirzhnits. 


\section{ACKNOWLEDGMENTS}

The author is grateful to G. Raffelt for drawing his attention to the paper by D.A. Kirzhnits and for helpful discussions and to the Max-Planck-Institut für Physik (WernerHeisenberg-Institut) for their hospitality. 


\section{REFERENCES}

[1] J.D. Jackson, Classical Electrodynamics (John Wiley, New York, 1975).

[2] M.H. Thoma, in Quark Gluon Plasma 2, edited by R. Hwa (World Scientific, Singapore, 1995).

[3] G.G. Raffelt, Stars at Laboratories for Fundamental Physics (University of Chicago Press, Chicago, 1996).

[4] R. Baier, D. Schiff, and B.G. Zakharov, hep-ph/0002198.

[5] E. Braaten and M.H. Thoma, Phys. Rev. D 44 (1991) 1298.

[6] J.I. Kapusta, Finite Temperature Field Theory (Cambridge University Press, New York, 1989).

[7] H. Schulz, Phys. Lett. B 291 (1992) 448.

[8] S. Ichimaru, Basic Principles of Plasma Physics, (Benjamin, Reading, 1973).

[9] M.H. Thoma and M. Gyulassy, Nucl. Phys. B 351 (1991) 491.

[10] H.T. Elze and U. Heinz, Phys. Rep. 183 (1989) 81.

[11] S. Mrówczyński, in Quark Gluon Plasma, edited by R. Hwa (World Scientific, Singapore, 1990).

[12] D.A. Kirzhnits, JETP Lett. 46 (1987) 308.

[13] M. Leontovich, Sov. Phys. JETP 13 (1961) 634.

[14] O.V. Dolgov, D.A. Kirzhnits, and V.V. Losyakov, Sov. Phys. JETP 56 (1982) 1095. 64, 331 (1994).

[15] E.M. Lifshitz and L.P. Pitajewski, Physikalische Kinetik (Akademie-Verlag, Berlin, 1986).

[16] E. Braaten and R.D. Pisarski, Nucl. Phys. B 337 (1990) 569.

[17] R.D. Pisarski, Physica A 158 (1989) 146.

[18] M.E. Carrington, H. Defu, and M.H. Thoma, Eur. Phys. J. C 7 (1999) 347.

[19] E. Braaten and M.H. Thoma, Phys. Rev. D 44 (1991) 2625.

[20] D.A. Kirzhnits, V.V. Losyakov, and V.A. Chechin, Sov. Phys. JETP 70 (1990) 609. 


\section{FIGURES}

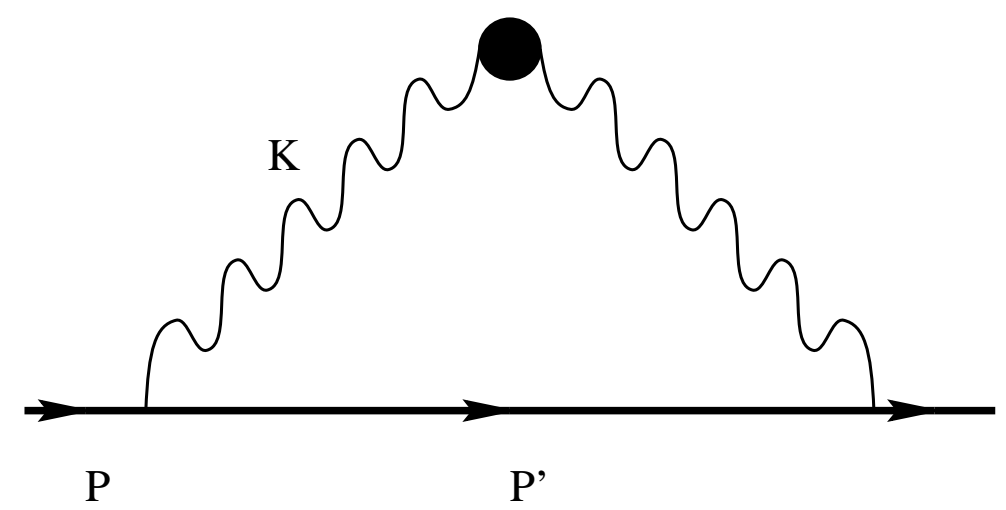

FIG. 1. Self energy of a fast fermion containing the full gauge boson propagator

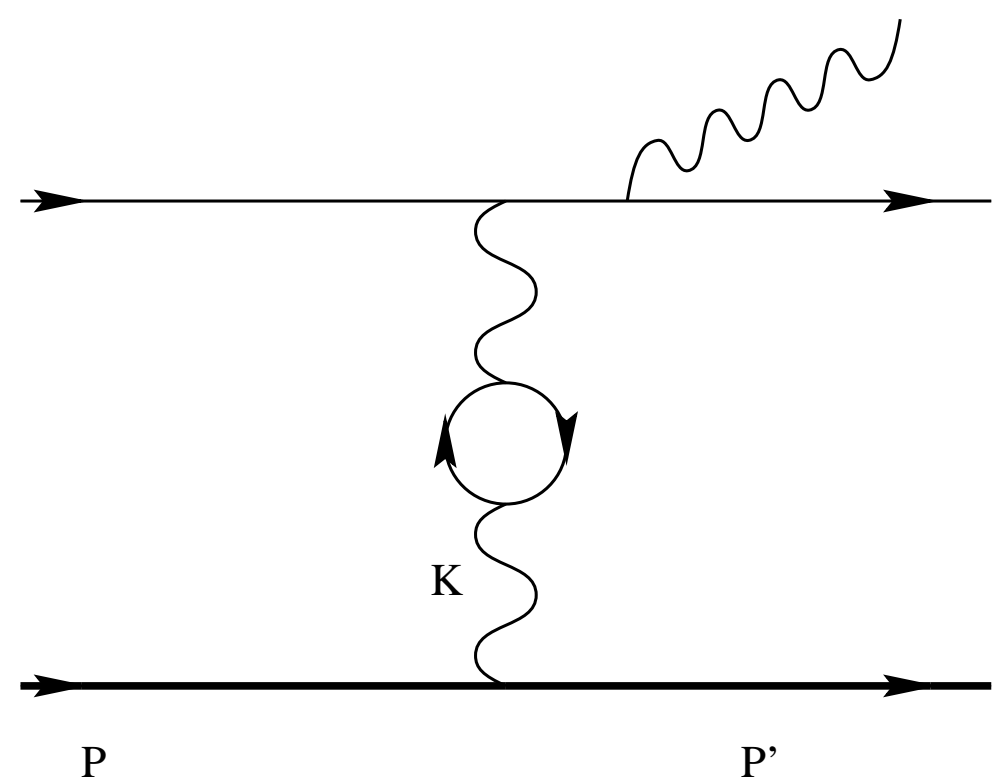

FIG. 2. Example for a scattering diagram related to the imaginary part of the diagram in Fig.1 